\title{
Boundedness of strong maximal functions with respect to non-doubling measures
}

\author{
Wei Ding ${ }^{1}$, LiXin Jiang ${ }^{2}$ and YuePing Zhu ${ }^{2 *}$
}

\author{
"Correspondence: \\ zhuyueping@ntu.edu.cn \\ ${ }^{2}$ Department of Mathematics, \\ Nantong Normal College, Nantong, \\ 226010, P.R. China \\ Full list of author information is \\ available at the end of the article
}

\begin{abstract}
The main purpose of this paper is to establish a boundedness result for strong maximal functions with respect to certain non-doubling measures in $\mathbb{R}^{n}$. More precisely, let $d \mu\left(x_{1}, \ldots, x_{n}\right)=d \mu_{1}\left(x_{1}\right) \cdots d \mu_{n}\left(x_{n}\right)$ be a product measure which is not necessarily doubling in $\mathbb{R}^{n}$ (only assuming $d \mu_{i}$ is doubling on $\mathbb{R}$ for $i=2, \ldots, n$ ), and let $\omega$ be a nonnegative and locally integral function such that $\omega_{i}(\cdot)=\omega\left(x_{1}, \ldots, x_{i-1}, \cdot, x_{i+1}, \ldots, x_{n}\right)$ is in $A_{\infty}^{1}\left(d \mu_{i}\right)$ uniformly in $x_{1}, \ldots, x_{i-1}, x_{i+1}, \ldots, x_{n}$ for each $i=1, \ldots, n-1$, let $d \nu=\omega d \mu, \nu(E)=\int_{E} \omega(y) d \mu(y)$, and $M_{\omega d \mu}^{n}$ be the strong maximal function defined by

$$
M_{\omega d \mu}^{n} f(x)=\sup _{x \in R \in \mathcal{R}} \frac{1}{v(R)} \int_{R}|f(y)| \omega(y) d \mu(y)
$$

where $\mathcal{R}$ is the collection of rectangles with sides parallel to the coordinate axes in $\mathbb{R}^{n}$. Then we show that $M_{\omega d \mu}^{n}$ is bounded on $L_{\omega d \mu}^{p}\left(\mathbb{R}^{n}\right)$ for $1<p<\infty$. This extends an earlier result of Fefferman (Am. J. Math. 103:33-40, 1981) who established the $L^{p}$ boundedness when $d \mu=d x$ is the Lebesgue measure on $\mathbb{R}^{n}$ and $d \nu=\omega d \mu$ is doubling with respect to rectangles in $\mathbb{R}^{n}, \omega$ satisfies a uniform $A_{\infty}^{1}$ condition in each of the variables except one.

Moreover, we also establish some boundedness result for the Cordoba maximal functions (Córdoba A. in Harmonic Analysis in Euclidean Spaces, pp. 29-50, 1978) associated with the Córdoba-Zygmund dilation in $\mathbb{R}^{3}$ with respect to some non-doubling measures. This generalizes the result of Fefferman-Pipher (Am. J. Math. 119:337-369, 1997).
\end{abstract}

Keywords: strong maximal functions; non-doubling measures; $A^{\infty}$ weights; reverse Hölder inequality; geometric covering lemmas; Córdoba's maximal functions; Córdoba-Zygmund dilations

\section{照 Springer}

\section{Introduction}

The classical theory of one-parameter harmonic analysis for maximal functions and singular integrals on $\left(\mathbb{R}^{n} ; \mu\right)$ has been developed under the assumption that the underlying measure $\mu$ satisfies the doubling property, i.e., there exists a constant $C>0$ such that $\mu(B(x ; 2 r)) \leq C \mu(B(x ; r))$ for every $x \in \mathbb{R}^{n}$ and $r>0$. However, some recent results [47] show that it should be possible to dispense with the doubling condition for most of the classical theory. It is well known that the use of doubling measure has two main advantages. One is that we can work with nested property. Another one is that the faces of

(c) Ding et al. 2016. This article is distributed under the terms of the Creative Commons Attribution 4.0 International License (http://creativecommons.org/licenses/by/4.0/), which permits unrestricted use, distribution, and reproduction in any medium, provided you give appropriate credit to the original author(s) and the source, provide a link to the Creative Commons license, and indicate if changes were made. 
the cubes have measure zero. As in $[4,6]$, we will only maintain the last property. If $\mu$ is a nonnegative Radon measure without mass-points, one can choose an orthonormal system in $\mathbb{R}^{n}$ so that any cube $Q$ with sides parallel to the coordinate axes satisfies the property $\mu(\partial Q)=0$ (Theorem 2 of [4]). The advantage of assuming this property is the continuity of the measure $\mu$ on cubes which can ensure that there is a Calderón-Zygmund decomposition $[4,6]$, which is one of the basic and most frequently used tools in the classical theory.

We first recall some well-known results on one-parameter $A_{p}(\mu)$ weights with respect to the possibly non-doubling measure $\mu$. We also refer to [8] for the general theory of classical weights. A $\mu$-measurable function $\omega$ is said to be a weight if it is nonnegative and $\mu$-locally integrable. A weight $\omega$ is said to be an $A_{p}(\mu)$ weight if $\omega$ satisfies the following definition.

Definition 1.1 Let $1<p<\infty$ and $p^{\prime}=p /(p-1)$. We say that a weight $\omega$ satisfies the $A_{p}(\mu)$ condition if

$$
\sup _{Q}\left(\frac{1}{\mu(Q)} \int_{Q} \omega d \mu\right)\left(\frac{1}{\mu(Q)} \int_{Q} \omega^{1-p^{\prime}} d \mu\right)^{p-1}<\infty,
$$

where sup is taken over all cubes whose sides are parallel to the coordinate axes.

We use the notation $A_{\infty}(\mu)=\bigcup_{p>1} A_{p}(\mu)$ to denote the class of weight functions $\omega \in$ $A_{p}(\mu)$ for some $p>1$.

When $\mu$ is a nonnegative Radon measure without mass-points, Lemma 2.3 in [6] tells us that some classical results for $\omega \in A_{p}(\mu)$ also hold. We state these results as follows.

Proposition A If $\mu$ is a nonnegative Radon measures in $\mathbb{R}^{n}$ without mass-points, for a weight $\omega$, the following conditions are equivalent:

(a) $\omega \in A_{\infty}(\mu)$;

(b) $\omega$ satisfies a reverse Hölder inequality; namely, there are positive constants $c$ and $\delta$ such that for every cube $Q$

$$
\left(\frac{1}{\mu(Q)} \int_{Q} \omega^{1+\delta} d \mu\right)^{1 /(1+\delta)} \leq \frac{c}{\mu(Q)} \int_{Q} \omega d \mu,
$$

and $c$ may be taken as close to 1 as $\delta \rightarrow 0$;

(c) there are positive constants $c$ and $\rho$ such that, for any cube $Q$ and any $\mu$-measurable set $F$ contained in $Q$,

$$
\frac{\omega(F)}{\omega(Q)} \leq c\left(\frac{\mu(F)}{\mu(Q)}\right)^{\rho}
$$

where $\omega(E)=\int_{E} \omega d \mu$;

(d) there are positive constants $\alpha, \beta<1$ such that whenever $F$ is a measurable set of a cube $Q$,

$$
\frac{\mu(F)}{\mu(Q)} \leq \alpha \quad \text { implies } \quad \frac{\omega(F)}{\omega(Q)} \leq \beta .
$$


Remark 1.1 The behavior of the constant $c$ in (b) is not explicitly obtained in [6]. But by a careful examination of its proof, we can find that $c$ may be chosen as $\left(1-\frac{\delta C_{0}}{C_{1}^{1+\delta}}\right)^{-1 /(1+\delta)}$ for two fixed constants $C_{0}, C_{1}$.

Let $\mathcal{B}_{x}$ be a collection of bounded sets containing $x \in \mathbb{R}^{n}$. Let $v$ be a positive measure. Given a locally integrable function $f$, denote

$$
\mathcal{M} f(x)=\sup _{R \in \mathcal{B}_{x}} \frac{1}{v(R)} \int_{R}|f(y)| d v(y) .
$$

If $\mathcal{B}_{x}$ is the collection of all the cubes containing $x \in \mathbb{R}^{n}$ (centered at $x$ ) whose sides are parallel to the coordinate axes, then we obtain the usual Hardy-Littlewood maximal function $M_{d v} f(x)$ with respect to the measure $d v$ (centered maximal function $\bar{M}_{d v} f(x)$ ). By means of the Besicovitch covering lemma, it is easy to prove that $\bar{M}_{d v}$ maps $L^{1}(d v)$ into weak $L^{1}(d v)$, and $L^{p}(d v)$ into $L^{p}(d v)$ for $p>1$. In dimension one, the non-centered maximal operator $M_{d v}$ is also shown to be bounded on $L^{p}(d v)$ for $p>1$ (see [9] and [10]). However, it is in general not true that $M_{d v} f(x)$ has these boundedness properties. We refer to [10] for counterexamples.

When $\mathcal{B}_{x}$ denotes the collection of all rectangles $R$ containing $x \in \mathbb{R}^{n}$ whose sides parallel to the coordinate axes, $\mathcal{M} \triangleq M_{d v}^{n}$ is the strong maximal operator with respect to measure $v$ in dimension $\mathrm{n}$. When $d v=d x$ is the Lebesgue measure on $\mathbb{R}^{n}$, Jessen, Marcinkiewcz and Zygmund [11] and Fava [12] showed that $M_{d x}^{n}$ is bounded on $L^{p}$ for all $p>1$. However, Fefferman [1] showed that it is generally not true for the boundedness properties for an arbitrary measure $d v$. It is thus natural to ask when is $M_{d v}^{n}$ bounded on $L^{p}(d v)$. Obviously, if $v$ on $\mathbb{R}^{n}$ is a product measure of $n$ one-dimensional nonnegative Radon measures, then the method of iteration works perfectly to show that $M_{d v}^{n}$ is bounded on $L^{p}(d v), 1<p<\infty$. For a general measure, the iteration method no longer works. In [1], Fefferman constructed a measure $v$ for which $M_{d v}^{n}$ is unbounded on $L^{p}(d v)$ for all $p<+\infty$, and gave a sufficient condition on $\omega$ for the $L^{p}(\omega d x)$ boundedness of $M_{\omega d x}^{n}$. Fefferman's result [1] can be stated as follows.

Theorem 1.1 Suppose that $d v(x)=\omega(x) d x$ on $\mathbb{R}^{n}$ where $\omega$ is a function which has the property of being uniformly in the class $A_{\infty}^{1}$ in each variable separately. Then $M_{d v}^{n}$ is a bounded operator on $L^{p}(d v)$ for all $1<p \leq \infty$.

In fact, the proof given in [1] also established a stronger result. This is given in Fefferman and Pipher [3].

Theorem 1.2 Suppose that $d v(x)=\omega(x) d x$ is a positive absolutely continuous measure on $\mathbb{R}^{n}$. Assume that $d v$ is doubling with respect to the family of all rectangles with sides parallel to the axes, and that $\omega$ is a function which has the property of being uniformly in the class $A_{\infty}^{1}$ in each variable separately except one. Then $M_{d v}^{n}$ is a bounded operator on $L^{p}(d v)$ for all $1<p \leq \infty$.

If we replace the Lebesgue measure $d x$ by a more general measure $d \mu$, which is not necessarily doubling, and use the notation $d \nu(x)=\omega(x) d \mu$, then it is not known whether the strong maximal function $M_{d \nu}^{n}$ is bounded on $L^{p}(d v)$ for all $1<p<\infty$. This is exactly 
one of the motivations of this paper. To this end, we first define the notion of $A_{p}$ weights with respect to the possibly non-doubling measure $d \mu$.

Definition 1.2 Let $1<p<\infty$ and $p^{\prime}=p /(p-1)$. We say that a weight $\omega$ satisfies the $\mathcal{A}_{p}(\mu)$ condition if

$$
[\omega]_{\mathcal{A}_{p}(\mu)}=\sup _{R \in \mathcal{B}}\left(\frac{1}{\mu(R)} \int_{R} \omega d \mu\right)\left(\frac{1}{\mu(R)} \int_{R} \omega^{1-p^{\prime}} d \mu\right)^{p-1}<\infty,
$$

where $\mathcal{B}$ is a collection of bounded sets.

Remark 1.2 If $\mathcal{B}$ is the collection of all rectangles whose sides are parallel to the coordinate axes, then we obtain product weights $\mathcal{A}_{p}(\mu) \triangleq A_{p}^{n}(\mu)$. If $d \mu\left(x_{1}, \ldots, x_{n}\right)=$ $d \mu_{1}\left(x_{1}\right) \cdots d \mu_{n}\left(x_{n}\right)$ is a product measure, then by the Lebesgue differentiation theorem, it is easy to see that, if $\omega \in A_{p}^{n}(\mu)$, then $\omega_{i}\left(x_{i}\right)=\omega\left(x_{1}, \ldots, x_{i-1}, \cdot, x_{i+1}, \ldots, x_{n}\right) \in A_{p}^{1}\left(\mu_{i}\right)$ uniformly, that is, there exists a constant $c>0$ such that, for a.e. $\left(x_{1}, \ldots, x_{i-1}, x_{i+1}, \ldots, x_{n}\right) \in \mathbb{R}^{n-1}$,

$$
\left[\omega_{i}\right]_{A_{p}^{1}\left(\mu_{i}\right)}=\sup _{I}\left(\frac{1}{\mu_{i}(I)} \int_{I} \omega_{i} d \mu_{i}\right)\left(\frac{1}{\mu_{i}(I)} \int_{I} \omega_{i}^{1-p^{\prime}} d \mu_{i}\right)^{p-1} \leq c<\infty,
$$

where the supremum is taken over all intervals $I$ in $\mathbb{R}$. Consequently $\omega_{i}$ satisfies the properties in Proposition A above uniformly in $x_{1}, \ldots, x_{i-1}, x_{i+1}, \ldots, x_{n}$ if $\mu_{i}$ is a Radon measure without mass-point.

If we replace the Lebesgue measure $d x$ by a product measure $d \mu(x)=d \mu_{1}\left(x_{1}\right) \times$ $d \mu_{2}\left(x_{2}\right) \times \cdots \times d \mu_{n}\left(x_{n}\right)$, inspired by the work of Fefferman [1], it is natural to ask the following.

Question 1 What conditions on $\omega(x)$ and $d \mu(x)$ can ensure the boundedness of the strong maximal function $M_{\omega d \mu}^{n}$ with respect to the measure $\omega d \mu$ on $L^{p}(\omega d \mu)$ for $1<p<\infty$ ?

Question 2 What conditions on $\omega(x)$ and $d \mu(x)$ can ensure the boundedness of the strong maximal function $M_{d \mu}^{n}$ with respect to the measure $d \mu$ on $L^{p}(\omega d \mu)$ for $1<p<\infty$ ?

If we work in $\mathbb{R}^{3}$ with the dilation group $\left\{\rho_{s, t}\right\}_{s, t>0}$ given by $\rho_{s, t}(x, y, z)=(s x, t z, s t z)$, that is $\mathcal{B}_{x}=$ the family of all rectangles containing $x \in \mathbb{R}^{3}$ whose sides are parallel to the coordinate axes in $\mathbb{R}^{3}$, and whose side lengths in the $x, y$, and $z$ directions are given by $s, t$, and $s \cdot t$ respectively (these rectangles are called Córdoba-Zygmund rectangles), then we get the Córdoba maximal function $\mathcal{M}(f)(x) \triangleq \mathbb{M}_{d v}(f)(x)$ with respect to the measure $d v$ whose sharp estimates have been obtained by Córdoba [2].

With the Córdoba-Zygmund rectangles, we can define Córdoba's weights $\mathcal{A}_{p}(\mu) \triangleq$ $\mathbb{A}_{p}(\mu)$. By the Lebesgue differential theorem, if $\omega \in \mathbb{A}_{p}(\mu)$, then $\omega(\cdot, y, z) \in A_{p}^{1}\left(\mu_{1}\right)$ uniformly in $y, z$, and $\omega(x, \cdot, z) \in A_{p}^{1}\left(\mu_{2}\right)$ uniformly in $x, z$.

When $d v=d x$, Fefferman [13] proved the following theorem.

Theorem 1.3 The weighted norm inequality

$$
\int_{\mathbb{R}^{3}}\left|\mathbb{M}_{d x}(f)(x)\right|^{p} \omega(x) d x \leq C \int_{\mathbb{R}^{3}}|f(x)|^{p} \omega(x) d x
$$


holds if and only if $\omega \in \mathbb{A}_{p}(d x)$.

Moreover, the following is proved by Fefferman and Pipher in [3].

Theorem 1.4 Suppose $d v=\omega(x, y, z) d x d y d z$ is a positive measure on $\mathbb{R}^{3}$ which is doubling with respect to all the Zygmund rectangles in $\mathbb{R}^{3}$ and uniformly in $A_{\infty}^{1}$ in the $x$ and $y$ variables. Then the Córdoba maximal function $\mathbb{M}_{d v}$ with respect to the measure dv is bounded on $L^{p}(d v)$ for all $1<p<\infty$.

When we replace the Lebesgue measure $d x d y d z$ by $d \mu(x, y, z)$ which is not necessarily doubling with respect to all the Córdoba-Zygmund rectangles in $\mathbb{R}^{3}$, it is then interesting to ask the following.

Question 3 Let $d \mu(x, y, z)$ be a nonnegative Radon measure on $\mathbb{R}^{3}$. What conditions on $\omega$ and $d \mu$ can guarantee the boundedness of the Córdoba strong maximal function $\mathbb{M}_{d \mu}(f)(x, y, z)$ with respect to the measure $d \mu$ on $L^{p}(\omega d \mu)$ for $1<p<\infty$ ?

Question 4 Let $d \nu(x, y, z)=\omega(x, y, z) d \mu(x, y, z)$ be a measure on $\mathbb{R}^{3}$. What conditions on $\omega$ and $d \mu$ can guarantee the boundedness of the Córdoba strong maximal function $\mathbb{M}_{d v}(f)(x, y, z)$ with respect to the measure $d v$ on $L^{p}(d v)$ for $1<p<\infty$ ?

In this paper, we always assume that $\mu=\mu_{1} \times \mu_{2} \times \cdots \times \mu_{n}$ is a product measure, where $\mu_{i}, i=1, \ldots, n$ are all nonnegative Radon measures without mass-points and complete. The assumption that $\mu_{i}$ are complete is just a technical requirement to allow us change the order of integration. For a rectangle $R \subseteq \mathbb{R}^{n}$, we mean a rectangle whose sides parallel to the coordinate axes.

The main theorems of this paper are as follows.

Theorem 1.5 Let $\mu(x)=\mu_{1}\left(x_{1}\right) \cdot \mu_{2}\left(x_{2}\right) \cdots \mu_{n}\left(x_{n}\right)$ be a product measure where $\mu_{i}$, $i=1, \ldots, n$ are all nonnegative Radon measures in $\mathbb{R}$ without mass-points and complete. Moreover, we assume that each $\mu_{i}$ for $2 \leq i \leq n$ is doubling on $\mathbb{R}$. If $\omega_{i}\left(x_{i}\right)=$ $\omega\left(x_{1}, \ldots, x_{i-1}, \cdot, x_{i+1}, \ldots, x_{n}\right) \in A_{\infty}^{1}\left(\mu_{i}\right)$ uniformly with respect to a.e. $\left(x_{1}, \ldots, x_{i-1}, x_{i+1}, \ldots, x_{n}\right) \in$ $\mathbb{R}^{n-1}$ for $i=1, \ldots, n-1$, then the operator $M_{\omega d \mu}^{n}$ is bounded on $L^{p}(\omega d \mu)$ for all $1<p<\infty$.

Theorem 1.6 Let $\mu(x)=\mu_{1}\left(x_{1}\right) \cdot \mu_{2}\left(x_{2}\right) \cdots \mu_{n}\left(x_{n}\right)$ be a product measure where $\mu_{i}, i=$ $1, \ldots, n$ are all nonnegative Radon measures in $\mathbb{R}$ without mass-points and complete. Moreover, we assume that each $\mu_{i}$ for $2 \leq i \leq n$ is doubling on $\mathbb{R}$. Then the strong maximal operator $M_{d \mu}^{n}$ with respect to the measure $d \mu$ is bounded on $L^{p}(\omega d \mu)$ if and only if $\omega \in A_{p}^{n}(d \mu)$ for all $1<p<\infty$.

Concerning the Córdoba maximal function associated with the Córdoba-Zygmund dilations $\rho_{s, t}$ in $\mathbb{R}^{3}$, when $\omega(x, y, z) d \mu(x, y, z)$ is not necessarily doubling with respect to all the Córdoba-Zygmund rectangles in $\mathbb{R}^{3}$, we have the following.

Theorem 1.7 Assume $\mu=\mu_{1} \times \mu_{2} \times \mu_{3}$, where $\mu_{2}, \mu_{3}$ are nonnegative Radon measures and satisfy the doubling property for all intervals $I \subseteq \mathbb{R}$, and $\mu_{1}$ is a nonnegative Radon 
measure in $\mathbb{R}$ without mass-points (which is not necessarily doubling). If $\omega \in \mathbb{A}_{p}(\mu)$, then the following weighted inequality holds:

$$
\int_{\mathbb{R}^{3}}\left[\mathbb{M}_{\omega d \mu}(f)(x, y, z)\right]^{q} \omega(x, y, z) d \mu(x, y, z) \leq C \int_{\mathbb{R}^{3}}|f(x, y, z)|^{q} \omega(x, y, z) d \mu(x, y, z),
$$

for all $1<q<\infty$.

Moreover, using Theorem 1.7 we have the following.

Theorem 1.8 Assume $\mu=\mu_{1} \times \mu_{2} \times \mu_{3}$, where $\mu_{2}, \mu_{3}$ are nonnegative Radon measures and satisfy the doubling property for all intervals $I \subseteq \mathbb{R}$, and $\mu_{1}$ is a nonnegative Radon measure in $\mathbb{R}$ without mass-points (which is not necessarily doubling). Then the Córdoba maximal operator $\mathbb{M}_{\mu}$ is bounded on $L^{p}(\omega d \mu)$ if and only if $\omega \in \mathbb{A}_{p}(\mu)$ for all $1<p<\infty$.

The organization of the paper is as follows. In Section 2, we will establish the reverse Hölder inequality for weights $\omega$ in the class $A_{p}^{n}(\mu)$ adapted to our general product measure $\mu$, which is not necessarily doubling with respect to the rectangles with sides parallel to the coordinate axes in $\mathbb{R}^{n}$. Section 3 gives the proofs of Theorems 1.5 and 1.6 of boundedness of strong maximal functions with respect to the non-doubling measures $d \mu$ and $d \nu=\omega d \mu$. In Section 4, we establish Theorems 1.7 and 1.8 for the Córdoba strong maximal functions with respect to the Córdoba-Zygmund dilations.

\section{Reverse Hölder inequality of weights $A_{p}^{n}(\mu)$}

The purpose of this section is to establish reverse Hölder inequality of weights in the class $A_{p}^{n}(\mu)$ adapted to our general product measure $\mu$.

We use the notation $w(E)=\int_{E} w(x) d \mu(x)$ for every measurable set $E \subset \mathbb{R}^{n}$ in this section.

Lemma 2.1 Assume $\mu$ is a nonnegative Radon measure. If $\omega \in A_{p}^{n}(\mu)$ for some $1<p<\infty$, then there exists $\eta>0$ such that whenever $F$ is a measurable subset of a rectangle $R$ and satisfies $\frac{\mu(F)}{\mu(R)} \leq \eta$, then

$$
\frac{\omega(F)}{\omega(R)} \leq 1-\eta
$$

Proof Since $\omega \in A_{p}^{n}(\mu)$ for some $1<p<\infty$, then when $f$ is non-negative and $L^{p}(\omega d \mu)$ integrable in a rectangle $R$, we have

$$
\begin{aligned}
\left(\int_{R} f d \mu\right)^{p} & =\left(\int_{R} f \omega^{\frac{1}{p}} \omega^{-\frac{1}{p}} d \mu\right)^{p} \\
& \leq\left(\int_{R} f^{p} \omega d \mu\right)\left(\int_{R} \omega^{-\frac{p^{\prime}}{p}} d \mu\right)^{\frac{p}{p^{\prime}}} \\
& \leq[\omega]_{A_{p}^{n}(\mu)}\left(\int_{R} f^{p} \omega d \mu\right) \frac{(\mu(R))^{p}}{\omega(R)}
\end{aligned}
$$

where $\frac{1}{p}+\frac{1}{p^{\prime}}=1$, which shows

$$
\left(\frac{1}{\mu(R)} \int_{R} f d \mu\right)^{p} \leq[\omega]_{A_{p}^{n}(\mu)} \frac{1}{\omega(R)} \int_{R} f^{p} \omega d \mu
$$


For a measurable set $F \subseteq R$, let $E=R \backslash F, f=\chi_{E}$, from the above inequality,

$$
\left(\frac{\mu(E)}{\mu(R)}\right)^{p} \leq[\omega]_{A_{p}^{n}(\mu)} \frac{\omega(E)}{\omega(R)}
$$

which implies

$$
\frac{\omega(F)}{\omega(R)} \leq 1-\frac{1}{[\omega]_{A_{p}^{n}(\mu)}}\left(1-\frac{\mu(F)}{\mu(R)}\right)^{p} .
$$

It is easy to see that, for a small enough $\eta>0$, when $\frac{\mu(F)}{\mu(R)}<\eta$, we have $1-\frac{1}{[\omega]_{A}^{n}(\mu)}\left(1-\frac{\mu(F)}{\mu(R)}\right)^{p}<$ $1-\eta$. Hence

$$
\frac{\omega(F)}{\omega(R)} \leq 1-\eta
$$

which completes the proof.

Remark 2.1 By the same proof, Lemma 2.1 holds for $\omega \in \mathbb{A}_{p}(\mu)$ with respect to the Córdoba-Zygmund rectangles in $\mathbb{R}^{3}$.

Since $[\omega]_{A_{p}^{n}(\mu)} \geq 1$, from the inequality (2.1), one can also easily obtain the following lemma.

Lemma 2.2 Under the same assumption on $\mu$ in Lemma 2.1, if $\omega \in A_{p}^{n}(\mu)$ for some $1<$ $p<\infty$, then, for all $0<\alpha<1$, there is a positive constant $\beta<1$ such that whenever $F$ is a measurable set of a rectangle $R$, we have

$$
\frac{\mu(F)}{\mu(R)} \leq \alpha \quad \text { implies } \quad \frac{\omega(F)}{\omega(R)} \leq \beta .
$$

This is equivalent to saying that, for all $0<\alpha^{\prime}<1$, there is a positive constant $\beta^{\prime}<1$ such that whenever $F$ is a measurable subset of a rectangle $R$,

$$
\frac{\mu(F)}{\mu(R)} \geq \alpha^{\prime} \quad \text { implies } \quad \frac{\omega(F)}{\omega(R)} \geq \beta^{\prime} .
$$

Remark 2.2 Equation (2.2) is called the $A_{\infty}^{n}(\mu)$ condition. It is easy to see that if $\omega \in$ $A_{p}^{n}(\mu), \omega_{i}\left(x_{i}\right)=\omega\left(x_{1}, \ldots, x_{i-1}, \cdot x_{i+1}, \ldots, x_{n}\right) \in A_{\infty}^{1}\left(\mu_{i}\right)$ uniformly with respect to $x_{1}, \ldots, x_{i-1}$, $x_{i+1}, \ldots, x_{n}$.

Under our assumption on $\mu$ being a product measure, we also have the reverse Hölder inequality for $\omega \in A_{p}^{n}(\mu)$.

Lemma 2.3 Assume that $\mu=\mu_{1} \times \mu_{2} \times \cdots \times \mu_{n}$ is a product measure, where the measures $\mu_{i}, i=1, \ldots, n$ are all nonnegative Radon measures without mass-points and complete. If $\omega \in A_{p}^{n}(\mu)$, for some $1<p<\infty$, then $\omega$ satisfies a reverse Hölder inequality, that is, there exist two positive constants $c$ and $\delta$ such that for every rectangle $R$

$$
\left(\frac{1}{\mu(R)} \int_{R} \omega^{1+\delta} d \mu\right)^{1 /(1+\delta)} \leq \frac{c}{\mu(R)} \int_{R} \omega d \mu,
$$


and c may be taken as close to 1 as $\delta \rightarrow 0^{+}$.

Proof By induction. When $n=1, \omega \in A_{p}^{1}(\mu)$, the result is obtained from Proposition A. Suppose that $n>1$, and this result holds for $n-1$. Denote $R=I_{1} \times I_{2} \times \cdots \times I_{n}=\tilde{I} \times I_{n}$, $\left(x_{1}, \ldots, x_{n}\right)=\left(p, x_{n}\right)$ and $\tilde{\mu}=\mu_{1} \mu_{2} \cdots \mu_{n-1}$. By the Lebesgue differentiation theorem, $\omega\left(\cdot, x_{n}\right)$ is uniformly in the class $A_{p}^{n-1}(\tilde{\mu})$, thus there exists $\delta_{1}>0$ such that

$$
\left(\frac{1}{\tilde{\mu}(\tilde{I})} \int_{\tilde{I}} \omega^{1+\delta_{1}}\left(p, x_{n}\right) d \tilde{\mu}\right)^{1 /\left(1+\delta_{1}\right)} \leq c \frac{1}{\tilde{\mu}(\tilde{I})} \int_{\tilde{I}} \omega\left(p, x_{n}\right) d \tilde{\mu}
$$

and $c$ may be taken as close to 1 as $\delta_{1} \rightarrow 0^{+}$.

Consider the function

$$
W\left(x_{n}\right)=\left(\frac{1}{\tilde{\mu}(\tilde{I})} \int_{\tilde{I}} \omega^{1+\delta_{1}}\left(p, x_{n}\right) d \tilde{\mu}(p)\right)^{1 /\left(1+\delta_{1}\right)} .
$$

We now prove that $W$ satisfies the reverse Hölder inequality. By Proposition A, we only need to prove that there are positive constants $\alpha, \beta<1$ such that, whenever $E$ is a measurable subset of interval $I_{n}$, one has

$$
\frac{\mu_{n}(E)}{\mu_{n}\left(I_{n}\right)} \leq \alpha \quad \text { implies } \quad \frac{W(E)}{W\left(I_{n}\right)} \leq \beta
$$

For a measurable subset $E \subset I_{n}$, by (2.3)

$$
\begin{aligned}
W(E) & =\int_{E} W\left(x_{n}\right) d \mu_{n}\left(x_{n}\right) \\
& \leq c \int_{E} \frac{1}{\tilde{\mu}(\tilde{I})} \int_{\tilde{I}} \omega\left(p, x_{n}\right) d \tilde{\mu}(p) d \mu_{n}\left(x_{n}\right) \\
& =c \frac{1}{\tilde{\mu}(\tilde{I})} \int_{E} \int_{\tilde{I}} \omega\left(p, x_{n}\right) d \tilde{\mu}(p) d \mu_{n}\left(x_{n}\right) \\
& =\frac{c}{\tilde{\mu}(\tilde{I})} \int_{F} \omega(x) d \mu(x),
\end{aligned}
$$

where $F=\tilde{I} \times E$. If $\frac{\mu_{n}(E)}{\mu_{n}\left(I_{n}\right)} \leq \alpha$, we have $\frac{\mu(F)}{\mu(R)}=\frac{\tilde{\mu}(\tilde{I}) \mu_{n}(E)}{\tilde{\mu}(\tilde{I}) \mu_{n}\left(I_{n}\right)} \leq \alpha$. Take $\alpha>0$ small enough, then $\omega(F)<(1-\alpha) \omega(R)$ by Lemma 2.1. So

$$
\begin{aligned}
W(E) & \leq \frac{c}{\tilde{\mu}(\tilde{I})} \omega(F) \\
& \leq \frac{c(1-\alpha)}{\tilde{\mu}(\tilde{I})} \int_{I_{n}} \int_{\tilde{I}} \omega\left(p, x_{n}\right) d \tilde{\mu}(p) d \mu_{n}\left(x_{n}\right) \\
& =c(1-\alpha) \int_{I_{n}} \frac{1}{\tilde{\mu}(\tilde{I})} \int_{\tilde{I}} \omega\left(p, x_{n}\right) d \tilde{\mu}(p) d \mu_{n}\left(x_{n}\right) \\
& \leq c(1-\alpha) \int_{I_{n}}\left(\frac{1}{\tilde{\mu}(\tilde{I})} \int_{\tilde{I}} \omega^{1+\delta_{1}}\left(p, x_{n}\right) d \tilde{\mu}(p)\right)^{1 /\left(1+\delta_{1}\right)} d \mu_{n}\left(x_{n}\right) \\
& =c(1-\alpha) W\left(I_{n}\right) .
\end{aligned}
$$


For a small enough $\alpha>0$, let $c$ close to 1 by taking $\delta_{1}$ close to $0^{+}$, we have $c(1-\alpha)<1$. Again, by Proposition A, $W$ satisfies the reverse Hölder inequality:

$$
\left(\frac{1}{\mu_{n}\left(I_{n}\right)} \int_{I_{n}} W^{1+\delta_{2}} d \mu_{n}\right)^{1 /\left(1+\delta_{2}\right)} \leq \frac{c^{\prime}}{\mu_{n}\left(I_{n}\right)} \int_{I_{n}} W d \mu_{n}
$$

and $c^{\prime}$ may be taken as close to 1 as $\delta_{2} \rightarrow 0$. Finally choosing $\delta_{1}=\delta_{2}=\delta$ sufficiently small, we have

$$
\begin{aligned}
& \left(\frac{1}{\mu(R)} \int_{R} \omega^{1+\delta} d \mu\right)^{1 /(1+\delta)} \\
& \quad=\left(\frac{1}{\mu(R)} \int_{R} \omega\left(p, x_{n}\right)^{1+\delta} d \tilde{\mu}(p) d \mu_{n}\left(x_{n}\right)\right)^{1 /(1+\delta)} \\
& \quad=\left(\frac{1}{\mu_{n}\left(I_{n}\right)} \int_{I_{n}}\left(\frac{1}{\tilde{\mu}(\tilde{I})} \int_{\tilde{I}} \omega\left(p, x_{n}\right)^{1+\delta} d \tilde{\mu}(p)\right)^{(1 /(1+\delta))(1+\delta)} d \mu_{n}\left(x_{n}\right)\right)^{1 /(1+\delta)} \\
& \quad=\left(\frac{1}{\mu_{n}\left(I_{n}\right)} \int_{I_{n}} W^{1+\delta} d \mu_{n}\right)^{1 /(1+\delta)} \\
& \quad \leq \frac{c^{\prime}}{\mu_{n}\left(I_{n}\right)} \int_{I_{n}} W d \mu_{n} \quad(\text { by }(2.4)) \\
& \left.\quad \leq c c^{\prime} \frac{1}{\tilde{\mu}(\tilde{I}) \mu_{n}\left(I_{n}\right)} \int_{R} \omega\left(p, x_{n}\right) d \tilde{\mu}(p) d \mu_{n}\left(x_{n}\right) \quad \text { (by }(2.3)\right)
\end{aligned}
$$

and from the above analysis, $c c^{\prime}$ may be taken as close to 1 by letting $\delta \rightarrow 0^{+}$. We then complete the proof.

If $\omega \in A_{p}^{n}(\mu), p>1$, then $\omega^{1-p^{\prime}} \in A_{p^{\prime}}^{n}(\mu)$, where $1 / p+1 / p^{\prime}=1$. Consequently, by Lemma 2.3 , it is easy to deduce the following corollary.

Corollary 2.1 Let $p>1$, and $\omega \in A_{p}^{n}(\mu)$, then there is an $\varepsilon>0$ such that $\omega \in A_{p-\varepsilon}^{n}(\mu)$.

Proof Since $\omega^{1-p^{\prime}} \in A_{p^{\prime}}^{n}(\mu)$ satisfies a reverse Hölder inequality for some exponent $\delta>0$ :

$$
\left(\frac{1}{\mu(R)} \int_{R} \omega^{\left(1-p^{\prime}\right)(1+\delta)} d \mu\right)^{1 /(1+\delta)} \leq \frac{c}{\mu(R)} \int_{R} \omega^{1-p^{\prime}} d \mu
$$

Fix $q$ such that $q^{\prime}-1=\left(p^{\prime}-1\right)(1+\delta)$. It is easy to see that $1<q<p$. By the above inequality, one has

$$
\begin{aligned}
& \left(\frac{1}{\mu(R)} \int_{R} \omega d \mu\right)\left(\frac{1}{\mu(R)} \int_{R} \omega^{1-q^{\prime}} d \mu\right)^{q-1} \\
& \quad \leq c\left(\frac{1}{\mu(R)} \int_{R} \omega d \mu\right)\left(\frac{1}{\mu(R)} \int_{R} \omega^{1-p^{\prime}} d \mu\right)^{(q-1)(1+\delta)},
\end{aligned}
$$

from which it follows that $\omega \in A_{q}^{n}(\mu)$ since $(q-1)(1+\delta)=p-1$ and $\omega \in A_{p}^{n}(\mu)$. Setting $\varepsilon=p-q$, we complete the proof. 


\section{The strong maximal functions with respect to non-doubling measures}

The main purpose of this section is to prove Theorem 1.5. We first need to prove the following geometric covering lemma whose proof is inspired by those in $[1,2,14]$, and [3] when $d u=d x$. Weak type estimates for strong maximal functions were first studied by Jessen, Marcinkiewcz and Zygmund [11] who first proved the strong differentiation theorem. Córdoba and Fefferman [14] gave a more geometric proof (see also Jawerth and Torchinsky [15]). Their method in [14] relies on a deep understanding of the geometry of rectangles. Namely, they established a deep and difficult geometric covering lemma. This lemma will lead to the weak type $(p, p)$ of $M_{\omega d \mu}^{n}$ as argued in [14]. Then we can complete the proof of Theorem 1.5 by interpolation (see, e.g., [16] and [17]). The proof of Theorem 1.6 is the same as that of Theorem 1.8 in Section 4, we shall omit it here.

Lemma 3.1 Assume that $\mu(x)=\mu_{1}\left(x_{1}\right) \cdot \mu_{2}\left(x_{2}\right) \cdots \mu_{n}\left(x_{n}\right)$ is a product measure where $\mu_{i}$, $i=1, \ldots, n$ are all nonnegative Radon measures in $\mathbb{R}$ without mass-points and complete. Assume also that each $\mu_{i}$ for $2 \leq i \leq n$ is doubling on $\mathbb{R}$ and that $\omega_{i} \in A_{\infty}^{1}\left(\mu_{i}\right)$ uniformly, $i=1, \ldots, n-1$.

Then, for all $1<p<\infty$, given a sequence $\left\{R_{i}\right\}$ of rectangles whose sides are parallel to the axes, there exists a subcollection $\left\{R_{i}^{*}\right\}$ such that

$$
\omega\left(\bigcup R_{i}\right) \leq c \omega\left(\bigcup R_{i}^{*}\right)
$$

and

$$
\left\|\sum \chi_{R_{i}^{*}}\right\|_{L^{p}(\omega d \mu)}^{p} \leq c \omega\left(\bigcup R_{i}^{*}\right) .
$$

Proof If we can prove it at $n=2$, then it is easy to complete the proof by induction. Hence we only give the proof when $n=2$.

With no loss of generality, we may assume $\left\{R_{i}\right\}$ is a finite sequence, and $R_{i}$ are arranged so that the side length in $x_{2}$ direction is decreasing. If $R=I \times J \subseteq \mathbb{R}^{2}$ is a rectangle, denote $\hat{R}=I \times 3 J$, where $3 J$ is an interval with the same center and three times the length of $J$. We choose $R_{1}^{*}=R_{1}$ and assume $R_{1}^{*}, \ldots, R_{k}^{*}$ have been selected. We obtain $R_{k+1}^{*}$ as the first rectangle $R$ on the list of $R_{i}$ after $R_{k}^{*}$ such that

$$
\mu\left(R \cap\left[\bigcup_{i \leq k, R \cap R_{i}^{*} \neq \emptyset} \hat{R}_{i}^{*}\right]\right)<\frac{1}{2} \mu(R) .
$$

We will prove that $\left\{R_{i}^{*}\right\}$ satisfies (3.1), (3.2). Now assume that some $R \in\left\{R_{i}\right\}$ was not selected, then we can find some positive integer $k$ such that

$$
\mu\left(R \cap\left[\bigcup_{i \leq k, R \cap R_{i}^{*} \neq \emptyset} \hat{R}_{i}^{*}\right]\right) \geq \frac{1}{2} \mu(R) .
$$

Let $I, I_{i}^{*}$ denote the slices of $R$ and $R_{i}^{*}$, respectively, with respect to hyperplanes perpendicular to the $x_{2}$. Since the sides of the rectangles $\left\{R_{i}\right\}$ parallel to the $x_{2}$ direction are in decreasing order, it is easy to obtain

$$
R \cap\left[\bigcup_{i \leq k, R \cap R_{i}^{*} \neq \emptyset} \hat{R}_{i}^{*}\right]=\left(I \cap\left[\bigcup_{i \leq k, R \cap R_{i}^{*} \neq \emptyset} \hat{I}_{i}^{*}\right]\right) \times J,
$$


where $\hat{R}_{i}^{*}=\hat{I}_{i}^{*} \times \hat{J}_{i}^{*}$. Then from (3.4) we have

$$
\mu_{1}\left(I \cap\left[\bigcup_{i \leq k} \hat{I}_{i}^{*}\right]\right) \geq \frac{1}{2} \mu_{1}(I) .
$$

Recalling that $\omega_{x_{2}}=\omega\left(\cdot, x_{2}\right) \in A_{\infty}^{1}\left(\mu_{1}\right)$ uniformly in $x_{2}$, by Remark 2.2 , there exists $0<\beta<1$, such that

$$
\omega_{x_{2}}\left(I \cap\left[\bigcup_{i \leq k} \hat{I}_{i}^{*}\right]\right) \geq \beta \omega_{x_{2}}(I)
$$

where $\omega_{x_{2}}(E)=\int_{E} \omega_{x_{2}}\left(x_{1}\right) d \mu_{1}\left(x_{1}\right)$ for a measure set $E \subseteq \mathbb{R}$, which implies that

$$
\bigcup_{i} I_{i} \subseteq\left\{x_{1} \mid M_{\omega_{x_{2}} d \mu_{1}}\left(\chi_{\cup \hat{I}_{i}^{*}}\right) \geq \beta\right\}
$$

For a one-dimensional Hardy-Littlewood maximal operator with respect to the measure $\omega_{x_{2}} d \mu_{1}$, it is well known that $M_{\omega_{x_{2}} d \mu_{1}}$ is bounded on $L^{2}\left(\omega_{x_{2}} d \mu_{1}\right)$ [10], which implies that

$$
\omega_{x_{2}}\left(\bigcup_{i} I_{i}\right) \leq c \omega_{x_{2}}\left(\bigcup \hat{I}_{i}^{*}\right)
$$

Integrating in $x_{2}$, we have

$$
\begin{aligned}
\omega\left(\bigcup_{i} R_{i}\right) & \leq c \omega\left(\bigcup_{i} \hat{R}_{i}^{*}\right) \\
& \leq c \sum_{i} \omega\left(\hat{R}_{i}^{*}\right) \\
& =c \sum_{i} \int_{I_{i}^{*}}\left(\int_{3)_{i}^{*}} \omega\left(x_{1}, x_{2}\right) d \mu_{2}\right) d \mu_{1} .
\end{aligned}
$$

By classical standard arguments, when $\mu_{2}$ is doubling, $\omega\left(x_{1}, \cdot\right) d \mu_{2}(\cdot)$ is doubling uniformly in $x_{1}$. Hence, we have

$$
\omega\left(\bigcup_{i} R_{i}\right) \leq c \sum_{i} \int_{I_{i}^{*}}\left(\int_{I_{i}^{*}} \omega\left(x_{1}, x_{2}\right) d \mu_{2}\right) d \mu_{1}=c \sum_{i} \omega\left(R_{i}^{*}\right) .
$$

Proceeding similarly to obtaining (3.5), by (3.3), for $\left\{R_{i}^{*}\right\}$ we have

$$
\mu_{1}\left(I_{k}^{*} \cap\left[\bigcup_{i<k, R \cap R_{i}^{*} \neq \emptyset} \hat{I}_{i}^{*}\right]\right)<\frac{1}{2} \mu_{1}\left(I_{k}^{*}\right),
$$

that is,

$$
\mu_{1}\left(I_{k}^{*} \cap\left[\bigcup_{i<k} \hat{I}_{i}^{*}\right]\right)=\mu_{1}\left(I_{k}^{*} \cap\left[\bigcup_{i<k} I_{i}^{*}\right]\right)<\frac{1}{2} \mu_{1}\left(I_{k}^{*}\right)
$$


By the assumption that $\omega_{x_{2}}$ is uniformly in $A_{\infty}^{1}\left(\mu_{1}\right)$ and by Proposition A, one obtains

$$
\omega_{x_{2}}\left(I_{k}^{*} \cap\left[\bigcup_{i<k} I_{i}^{*}\right]\right) \leq \beta^{\prime} \omega_{x_{2}}\left(I_{k}^{*}\right)
$$

for some $\beta^{\prime}<1$. Let $E_{k}=I_{k}^{*} \backslash\left[\bigcup_{i<k} I_{i}^{*}\right]$, then $\omega_{x_{2}}\left(E_{k}\right) \geq\left(1-\beta^{\prime}\right) \omega_{x_{2}}\left(I_{k}^{*}\right)$. So

$$
\begin{aligned}
\omega\left(\bigcup_{i} R_{i}\right) & \leq c \sum_{i} \omega\left(R_{i}^{*}\right) \\
& =c \sum_{i} \int_{J_{i}^{*}}\left(\int_{I_{i}^{*}} \omega\left(x_{1}, x_{2}\right) d \mu_{1}\right) d \mu_{2} \\
& \leq c /\left(1-\beta^{\prime}\right) \sum_{i} \int_{J_{i}^{*}}\left(\int_{E_{i}} \omega\left(x_{1}, x_{2}\right) d \mu_{1}\right) d \mu_{2} \\
& =c /\left(1-\beta^{\prime}\right) \int_{\bigcup J_{i}^{*} \times E_{i}} \omega\left(x_{1}, x_{2}\right) d \mu \\
& \leq c /\left(1-\beta^{\prime}\right) \omega\left(\bigcup_{i} R_{i}^{*}\right),
\end{aligned}
$$

which leads to (3.1).

Finally, as done in $[1,3]$ by duality, we assume that $\varphi$ is a function on $\mathbb{R}$ satisfying $\|\varphi\|_{L^{p^{\prime}}\left(\omega_{x_{2}} d \mu_{1}\right)}=1, \frac{1}{p}+\frac{1}{p^{\prime}}=1$, then by the $L^{p^{\prime}}\left(\omega_{x_{2}} d \mu_{1}\right)$ boundedness of $M_{\omega_{x_{2}}} d \mu_{1}$ again, one has, for a.e. $x_{2}$,

$$
\begin{aligned}
\int \sum \chi_{I_{k}^{*}} \varphi \omega_{x_{2}} d \mu_{1} & =\sum \int_{I_{k}^{*}} \varphi \omega_{x_{2}} d \mu_{1} \\
& =\sum\left(\frac{1}{\omega_{x_{2}}\left(I_{k}^{*}\right)} \int_{I_{k}^{*}} \varphi \omega_{x_{2}} d \mu_{1}\right) \omega_{x_{2}}\left(I_{k}^{*}\right) \\
& \leq \sum \frac{1}{1-\beta^{\prime}} \omega_{x_{2}}\left(E_{k}\right) \inf _{x_{1} \in I_{k}^{*}} M_{\omega_{x_{2}}} d \mu_{1}(\varphi)\left(x_{1}\right) \\
& \leq \frac{1}{1-\beta^{\prime}} \int_{\bigcup I_{k}^{*}} M_{\omega_{x_{2}} d \mu_{1}}(\varphi) \omega_{x_{2}} d \mu_{1} \\
& \leq \frac{1}{1-\beta^{\prime}}\left(\int_{\bigcup I_{k}^{*}} M_{\omega_{x_{2}}}^{p^{\prime}} d \mu_{1}(\varphi) \omega_{x_{2}} d \mu_{1}\right)^{1 / p^{\prime}}\left(\omega_{x_{2}}\left(\bigcup I_{k}^{*}\right)\right)^{1 / p} \\
& \leq c\left(\omega_{x_{2}}\left(\bigcup I_{k}^{*}\right)\right)^{1 / p},
\end{aligned}
$$

which implies

$$
\int\left(\sum \chi_{I_{k}^{*}}\right)^{p} \omega_{x_{2}} d \mu_{1} \leq c \omega_{x_{2}}\left(\bigcup I_{k}^{*}\right) .
$$

Integrating over $x_{2}$ finishes the proof of (3.2).

\section{Córdoba's maximal function}

In this section, as an application of Theorem 1.5, we study the necessary and sufficient conditions on $\omega$ for the weighted inequality for the Córdoba maximal function $\mathbb{M}_{\mu}(f)$ in 
$\mathbb{R}^{3}$ with respect to the not necessarily doubling measure $\mu$ :

$$
\int_{\mathbb{R}^{3}}\left[\mathbb{M}_{\mu}(f)(x, y, z)\right]^{p} \omega(x, y, z) d \mu(x, y, z) \leq C \int_{\mathbb{R}^{3}}|f(x, y, z)|^{p} \omega(x, y, z) d \mu(x, y, z) .
$$

Proof of Theorem 1.7 Using the fact that $\omega \in \mathbb{A}_{p}(\mu)\left(A_{p}\right.$ weights with respect to the Córdoba-Zygmund rectangles and the not necessarily doubling measure $\mu$ ), we see that $w(\cdot, y, z)$ is in $A_{p}^{1}\left(d \mu_{1}\right)$ uniformly in $y, z$ and $w(x, \cdot, z)$ is in $A_{p}^{1}\left(d \mu_{2}\right)$ uniformly in $x, z$. By the assumptions that the measures $\mu_{2}, \mu_{3}$ are doubling on $\mathbb{R}$, Theorem 1.7 is an immediate corollary of Theorem 1.5 .

We will prove Theorem 1.4 by an argument similar to the one given in [13]. We first prove the reverse Hölder's inequality for $\omega \in \mathbb{A}_{p}(\mu)$. For a fixed number $a>0$, let $\mathcal{U}$ be the family of all rectangles whose sides are parallel to the coordinate axes in $\mathbb{R}^{2}$, and whose side lengths in the $x, y$ directions are given by $s$ and $s a$, where $s$ is arbitrary. First of all, by Corollary 9.2.4 of [18], using a linear change of scale we obtain the following proposition.

Proposition 4.1 Let $v=v_{1} \times v_{2}$ be a product measure, where $v_{1}$, $v_{2}$ are nonnegative Radon measures and satisfy the doubling property for all interval $I \subseteq \mathbb{R}$, and $\omega$ be a weight. Suppose that there exist $0<\alpha, \beta<1$ such that, for $\forall R \in \mathcal{U}$,

$$
\frac{v(F)}{v(R)} \leq \alpha \quad \text { implies } \quad \int_{F} \omega d v \leq \beta \int_{R} \omega d v
$$

where $F$ is a $v$-measurable subset of $R$. Then there are positive constants $c$ and $\gamma$ such that for every rectangle $R \in \mathcal{U}$

$$
\left(\frac{1}{v(R)} \int_{R} \omega^{1+\gamma} d \nu\right)^{1 /(1+\gamma)} \leq \frac{c}{v(R)} \int_{R} \omega d \nu
$$

Proceeding as in [13] or the proof of Lemma 2.3, together with the above proposition, we can establish the following reverse Hölder inequality for Córdoba's weights with respect to a certain non-doubling measure $\mu$. We omit the proof.

Proposition 4.2 There are positive constants $c$ and $\varepsilon$ such that, for all Córdoba-Zygmund rectangles $R$

$$
\left(\frac{1}{\mu(R)} \int_{R} \omega^{1+\varepsilon} d \mu\right)^{1 /(1+\varepsilon)} \leq \frac{c}{\mu(R)} \int_{R} \omega d \mu
$$

Then with a proof similar to that of Corollary 2.1, one has the following result.

Corollary 4.1 Let $p>1$, and $\omega \in \mathbb{A}_{p}(\mu)$. Then there is an $\varepsilon>0$ such that $\omega \in A_{p-\varepsilon}(\mu)$.

We are now ready to complete the proof of Theorem 1.8.

Proof of Theorem 1.8 The necessity follows just as in the classical case. Now if $\omega \in \mathbb{A}_{p}(\mu)$, by Hölder's inequality, one has

$$
\mathbb{M}_{\mu}(f) \leq[\omega]_{\mathbb{A}_{p}(\mu)}\left[\mathbb{M}_{\omega d \mu}\left(|f|^{p}\right)\right]^{1 / p}
$$


Then $\mathbb{M}_{\mu}$ is $L^{q}(\omega d \mu)$ bounded for every $q>p$ by Theorem 1.7. Using the fact $\omega \in A_{p-\varepsilon}(\mu)$ for some $\varepsilon>0$ by Corollary 4.1 , one sees that $\mathbb{M}_{\mu}$ is bounded on $L^{p}(\omega d \mu)$.

\section{Competing interests}

The authors declare that they have no competing interests.

\section{Authors' contributions}

All the authors contributed equally and significantly in writing this paper. All the authors read and approved the final manuscript.

\section{Author details}

${ }^{1}$ School of Sciences, Nantong University, Nantong, 226007, P.R. China. ${ }^{2}$ Department of Mathematics, Nantong Normal College, Nantong, 226010, P.R. China.

\section{Acknowledgements}

The authors would like to thank the referees very much for the kind advice and useful suggestions.

Article is supported by NNSF of China grants $(11501308,11271209)$

Received: 4 March 2016 Accepted: 8 November 2016 Published online: 22 November 2016

\section{References}

1. Fefferman, R: Strong differentiation with respect to measures. Am. J. Math. 103, 33-40 (1981)

2. Córdoba, A: Maximal functions, covering lemmas and Fourier multipliers. In: Harmonic Analysis in Euclidean Spaces. (Proc. Sympos. Pure Math., Williams Coll, Williamstown, Mass, 1978) Part 1, Proc. Sympos. Pure Math., vol. XXXV, pp. 29-50. Am. Math. Soc., Providence, Rl (1978)

3. Fefferman, R, Pipher, J: Multiparameter operators and sharp weighted inequalities. Am. J. Math. 119, 337-369 (1997)

4. Mateu, J, Mattila, P, Nicolau, A, Orobitg, J: BMO for nondoubling measures. Duke Math. J. 102, 533-565 (2000)

5. Nazarov, F, Treil, S, Volberg, A: Cauchy integral and Calderón-Zygmund operators on nonhomogeneous spaces. Int. Math. Res. Not. 15, 703-726 (1997)

6. Orobitg, J, Pérez, C: $A_{p}$ weights for nondoubling measures in $R^{n}$ and applications. Trans. Am. Math. Soc. 354 2013-2033 (2002)

7. Tolsa, X: BMO, $H^{1}$ and Calderón-Zygmund operators for non doubling measures. Math. Ann. 319, 89-149 (2001)

8. Garcia-Cuerva, J, Rubio de Francia, J: Weighted Norm Inequalities and Related Topics. North-Holland Mathematics Studies, vol. 116. North-Holland, Amsterdam (1985)

9. Muckenhoupt, B, Stein, EM: Classical expansions and their relation to conjugate harmonic functions. Trans. Am. Math Soc. 118, 17-92 (1965)

10. Sjögren, P: A remark on maximal function for measures in $R^{n}$. Am. J. Math. 105(5), 1231-1233 (1983)

11. Jessen, B, Marcinkiewcz, J, Zygmund, A: Note on the differentiability of multiple integrals. Fundam. Math. 25, 217 (1935)

12. Fava, N: Weak type inequalities for product operators. Stud. Math. 42, 271-288 (1972)

13. Fefferman, R: Some weighted norm inequalities for Córdoba's maximal function. Am. J. Math. 106(5), 1261-1264 (1984)

14. Córdoba, A, Fefferman, R: A geometric proof of the strong maximal theorem. Ann. Math. 102(1), 95-100 (1975)

15. Jawerth, B, Torchinsky, A: The strong maximal function with respect to measures. Stud. Math. 80(3), 261-285 (1984)

16. Grafakos, L: Classical Fourier Analysis. Springer, Berlin (2008)

17. Stein, EM: Harmonic Analysis: Real-Variable Methods, Orthogonality, and Oscillatory Integrals. Princeton Mathematical Series, vol. 43. Monographs in Harmonic Analysis, III. Princeton University Press, Princeton (1993)

18. Grafakos, L: Modern Fourier Analysis. Springer, Berlin (2008)

\section{Submit your manuscript to a SpringerOpen ${ }^{\ominus}$ journal and benefit from:}

- Convenient online submission

Rigorous peer review

- Immediate publication on acceptance

- Open access: articles freely available online

- High visibility within the field

- Retaining the copyright to your article 